\title{
Biomarkers for development of cancer vaccines
}

\section{Mike Whelan', Graham Ball2, Chris Beattie \& Angus D algleish ${ }^{4 \dagger}$}

${ }^{\dagger}$ Author for correspondence ${ }^{1}$ St G eorgés H ospital M edical School,

Onywax Ltd,

Cranmer Terrace,

London, SW 17 ORE, UK

Tel.: +44 2086829109 ;

Fax: +44 208682 9495;

E-mail.mwhelan@

onyax.com

${ }^{2} \mathrm{~N}$ ottingham Trent

University,

School of Biomedical and

$\mathrm{N}$ atural Sciences,

Clifton Campus,

Clifton Lane, Nottingham,

N G11 8N S, UK

${ }^{3} \mathrm{St}$ Georgés H ospital M edical School,

Onywax Ltd,

Cranmer Terrace, London,

SW 17 ORE, UK

${ }^{4} \mathrm{St} \mathrm{G}$ eorgés H ospital M edical School,

D epartment of O ncology, Cranmer Terrace, London, SW 17 ORE, UK

Tel.: +44 0181725 8815; Fax: +44 0181725 2992;

E-mail: dalgleis@sgul.ac.uk

Keywords: biomarkers, cancer vaccines, clinical trials, immunotherapy

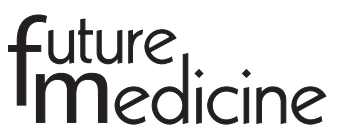

\begin{abstract}
The search for molecules that correlate with cancer progression is an evolving and exciting area of research. Such biomarkers have utility in a number of areas, most notably in a variety of clinical development programs. Modern technologies are revealing multiple potential biomarker candidates. However, the challenge remains in validating the correlation in levels of a particular molecule with clinical parameters. In this article, the area of biomarkers and cancer vaccines is briefly reviewed and the possibility of combining multiple molecules to generate a profile that correlates with outcome, rather than using more conventional single molecule biomarker systems, is explored.
\end{abstract}

The term biomarker refers to any biological indicator that reflects disease status but may not necessarily be involved in the disease process itself. $\mathrm{N}$ ot all biomarkers are surrogate markers, since the latter have to be statistically proven to correlate with a clinical end point, such as overall survival (OS) [1]. Biomarkers vary from being highly disease and stage specific, for example, prostate specific antigen (PSA) [2], to the nonspecific parameters of measuring inflammation that may correlate with an increased risk of a number of diseases, including ischemic heart disease and cancer. O ne such example is $\mathrm{C}$-reactive protein (CRP) [3], which is associated with increased coronary artery disease when it is continually elevated.

The number of potential biomarkers is increasing daily with the advent of genomics, proteomics, metabolomics and other sophisticated imaging technologies. M ass spectroscopy, two-dimensional gels and gene arrays, in particular, are producing hundreds of new candidates, with additional scope for improvement of these technologies. However, not all of these newly identified molecules will become useful biomarkers and it is an obligation of researchers to demonstrate a meaningful correlation between disease state and molecular levels before any of these may be used in the clinical setting.

Biomarkers are of great use in both clinical research and drug or vaccine development, if properly validated. They can aid patient selection, by allowing stratification and risk assessment, and can also predict treatment responses based on the expression of surrogate markers. Clinical end points, such as $\mathrm{OS}$ and time to disease progression (TTP), which itself is a surrogate for survival in some conditions, can require very long and expensive clinical trial protocols. Accurate biomarkers could greatly reduce the time needed to assess if a drug has significant clinical activity in Phase II trials before progressing to larger, and more costly, Stagelll trials.

There are many good cancer biomarker candidates which are regularly used by clinicians but have not necessarily been validated for clinical trial use. Well-known examples include $\alpha$ fetoprotein $(\alpha F P)$ and human chorionic gonadotropin (HCG) in chorionic and testicular cancers [4], CA125 in ovarian cancers [5], CA19.9 in pancreatic cancer [6], carcinoma embryonic antigen (CEA) in colorectal cancer [7], and PSA in prostate cancers [2]. $M$ any of these markers are not necessarily limited to the indicated tumor type and may be elevated in a number of other cancers, although, admittedly, not as regularly as the above mentioned associations. However, where tumors are caused by genetic lesions, such as is seen in chronic myeloid leukemia $(C M L)$, the use of polymerase chain reaction (PCR) can lead to the development of highly specific biomarkers, with PCR negativity confirming total disease elimination [8]. If similar molecular targets could be identified for other diseases, these could prove extremely useful as biomarkers based on specific molecular abnormalities.

Biomarkers are particularly needed to screen the new generation of drugs that are more likely to slow the rate of progression of disease than to induce complete responses. Examples of these include Avastin ${ }^{\circledR}$ (bevacizumab) [9] and $\operatorname{Tarceva}^{\circledR}$ (erlotinib) [10], although cancer vaccines also fall into this category. To date, most cancer vaccines do not usually induce dramatic tumor reductions. Those most likely to benefit are patients with totally resected disease with a high risk of relapse, such as Stage III melanoma, or in slowly progressive diseases with minimal tumor volume. Biomarkers 
would be of obvious advantage in assessing the hundreds of different treatment protocols that have been used to date.

In addition to disease-specific biomarkers, there are immune response biomarkers that may be of use in identifying patients who are mounting a meaningful clinical response at an early stage. Clearly, it would be advantageous to select these patients as early as possible, and also to move nonresponding patients onto alternative treatment modalities. Examples of such immunological markers include cytokine production, antibody induction against tumor-associated antigens and cytotoxic T-lymphocytes (CTL) activity against tumor cells.

In this review, we use the example of prostate cancer (Box 1), which has been targeted by a number of different vaccine candidates with early encouraging results, to demonstrate how novel immunological parameter measurements can be combined to develop biomarker patterns. Rather than using single molecules, it has been found to be particularly useful to combine several markers together, thus making a profile, rather than the more conventional linear relationship between a single molecule and disease.

\section{Specific immunotherapies}

The choice of immunotherapies available has increased vastly in recent years, ranging from single peptide vaccinations to whole tumor cell therapies. H owever, not all of these are appropriate for every patient, and the use of biomarkers may well help in ascribing particular therapies to an individual. O ne such variation in patient phenotype suggests that regulatory $\mathrm{T}$-cells play a significant role in downregulating immune responses and, therefore, attempts have been made to deplete them before any therapy is administered [11]. Some of the more common immunotherapy approaches, for multiple tumor types, are discussed below.

\section{Peptide vaccination}

Although direct evidence is scarce, it is widely believed that T-cell immunity is vital for a viable immunotherapeutic approach. As part of the activation of $C D \& C T L$, antigens are broken down from their constituent proteins to peptides [12]. Therefore, it follows that vaccination of patients using peptides derived from prostate antigens may lead to the beneficial generation of protective immunity. This approach has undergone several rounds of refinement ranging from single peptide vaccination to more complex mixtures containing several potentially useful epitopes [13]. Recent studies have also shown that more complete $\mathrm{T}$-cell activation may be achieved when at least one CD 4 helper T-cell epitope is included al ongside the CD 8 targets $[14,15]$.

\section{Nucleic acid vaccination}

This may be thought of as an extension of peptide therapy, although the mode of antigen delivery is substantially different. The two main routes used are either naked DNA injection [16,17], or the use of a viral vector to carry a gene of target antigen [18]. In the former, DNA is used to encode either single epitopes or larger fragments of a target antigen. This is injected intramuscularly and transcribed into immunogenic protein. In turn, this leads to the generation of strong immune responses. In the latter strategy, a virus is designed to carry the target epitope that will then infect a cell and lead to the production of the peptide in the host cytoplasm. Effectively, this will deliver the epitope into the human leukocyte antigen ( $\mathrm{HLA}$ ) class I antigen processing pathway and should, therefore, lead to CT L priming.

Recombinant antigens

Although this mode of immunization predates peptide vaccination, it is effectively a subset, since the protein must still be broken down into its constituent peptides before presentation to the immune system. Interestingly, this area has undergone somewhat of a resurgence in the wake of the poor clinical responses recorded after peptide vaccination. This modality has several advantages, including not being limited to patients carrying a particular HLA allele, since the immune system can select relevant peptides which will bind to an individual's own HLA molecules, and due to the relative ease of manufacturing pure recombinant products [19].

Dendritic cell therapy

The dendritic cell (DC) is a professional antigenpresenting cell and is capable of initiating primary immune responses. Consequently, much attention has focused on the utilization of $D C$ as a vaccine against cancer. As discussed previously, antigen processing is necessarily H LA-restricted and therefore $D C$ therapies are true personalized medicines since each batch of vaccine will be patient specific. Large-scale clinical trials using autologous DC loaded with PSA and granulocyte monocytecolony stimulating factor (GM-CSF) are ongoing and some initial clinical data are encouraging [20,21]. An alternative 


\section{Box 1. Prostate cancer.}

Prostate cancer remains a major cause of male death, and advanced hormone refractory prostate cancer (HRPC) has a poor prognosis, for which, until recently, no therapeutic treatments were available. Taxotere has shown some survival activity but is usually reserved for symptomatic disease [41]. Asymptomatic individuals often still possess an intact immune system, and hence immunotherapy is a viable option. Whilst the principle that the immune system is capable of controlling cancer is well established [38,42-44], it is only in recent years that advances in immunotherapy have begun to show real clinical benefit [30,45]. Although this is attractive, pitfalls still exist, since it is important to identify patients with fully active immune systems to give them both the optimal chance of mounting a meaningful response and also to avoid subjecting them to treatments with a low probability of success. Hence, there is a pressing need to identify useful biomarkers that will allow rapid discrimination of clinically responding and nonresponding individuals.

Given the disparate nature of the extremely complex biological events that occur during such therapy, it is particularly challenging to correlate immune response with clinical benefit. In this article, the authors attempt to review this dilemma and use their own experiences of whole-cell vaccination as a paradigm of how such issues may be addressed.

In established prostate cancer, transient rises in prostate-specific antigen (PSA) may be due to other causes, such as the PSA flare associated with cessation of hormonal therapies, and in general, rising levels of PSA indicate advancing disease. In clinical practice, PSA rises are generally accepted to be a sign of disease progression and may trigger new or additional treatment interventions, including use of investigational products [46]. Thus, PSA increases have commonly been used in early phase clinical trials as a surrogate for disease progression, while reductions in absolute PSA levels have been used in such studies as a surrogate for clinical efficacy [2]. Despite this, PSA is not acceptable as a clinical trial end point for regulatory approvals [47]. One reason for this is that despite being able to confirm the association between PSA and overall survival (OS) at an individual patient level, neither prospective nor retrospective analyses have been able to establish a high correlation between the treatment effects on PSA and the true clinical end point (OS) across two or more interventions [45]. Furthermore, recent data suggest that screening PSA levels are a poor indicator of tumor burden in either black individuals or individuals older than 62 years [48].

Refinements of PSA monitoring methodologies, such as measuring PSA velocity (PSAV), show promise as more clinically relevant biological endpoints. How ever, these too have yet to achieve acceptance with regulatory authorities as definitive biomarkers [46].

approach has been pioneered by Su and colleagues, in which autologous DC S are transfected with tumor-derived RN A. Although still in early phase trials, this has shown some immunological activity [22].

\section{Whole-cell vaccines}

A logical extension of single epitope vaccination is to employ a replication incompetent tumor cell as a vaccine. This is perhaps the most analogous system to true 'Jenner' vaccination, since it should be applicable to all individuals. The vaccine will be broken down into individual peptides and then presented in the context of the patient's own HLA molecules, be that one, or both, of the class I and class II systems. Such an approach is attractive, as it ensures that multiple antigens are delivered simultaneously, thus increasing the probability that clinically relevant immune responses are generated.

Wholecell vaccine approaches are generally divided into two categories; autologous and allogeneic. In the former, the patient's own tumor is used as the source of vaccine. This is a highly personalized therapy and requires the generation of either short-term cultures or fully characterized cell lines derived from the tumor. This has been attempted in a number of tumors including melanoma [23,24] and sarcoma [25]. This has variable success rates and does pose the question: why should an autologous vaccine elicit an effective immune response by the patient, when the tumor, in situ, did not? Clearly, there are differences between actively growing tumors and replication incompetent vaccines. However, observations in the author's own laboratories suggest that the antigenic profile is remarkably conserved. Activation is usually achieved by the introduction of adjuvants and by the altered phenotype of the resulting cell line. However, the question of overcoming host tolerance is fundamental to all vaccine approaches.

The second approach is to use allogeneic whole cell vaccines. It is well established that tumors from a variety of sources share multiple antigens. Examples include the melanoma antigen gene (M AGE), $\mathrm{N}$ ew York esophageal squamous cell carcinoma 1 (N Y-ESO 1) and mucin 1 (M U C1) $[26,27]$. Given that the proposed mechanism of action requires that the vaccine will be broken down into its constituent peptides, it follows that the H LA type of the vaccine is of secondary importance, since antigen processing will occur regardless of the patient's tissuetype. Indeed, it could be argued that an HLA mismatch might actually be a more potent vaccine because the host versus graft response, initiated by the presence of 'wrong' H LA molecules, may amplify the reaction [28]. Unfortunately, the encouraging Phase II trials with Canvaxin ${ }^{\mathrm{TM}}$ in melanoma [29] have not been followed by positive 
late stage data with very recent failures in a large Phase III melanoma trial [101]. It is interesting to note that this worldwide trial was randomized against bacillus Calmette-Guerin (BCG), which is known to haveimmunological activity although its direct clinical effect in melanoma is variable [30,31]. Similarly, M elacine ${ }^{\circledR}$ failed to show significant efficacy in a randomized trial [32], although further analysis demonstrated that patients with either the HLA-A2 or HLA-C3 class I alleles did show a survival benefit [33].

\section{Immunological monitoring}

The use of vaccines allows immune parameters to be employed as potential surrogates for monitoring beneficial clinical responses. H owever, perhaps the most common pitfall in this entire field of endeavor is the measurement of relevant biomarkers. Indeed, it may even be the case that the response generated is quite different from the one expected. The presence of a specific immune response does not necessarily correlate with clinical outcome. In a recent melanoma trial using a peptide specific for an epitope in MAGE-3 presented by $H L A-A 1$, it was found that the most important response was to a completely different peptide, derived from M AGE-C2 and presented by HLA-A2 [34,35]. Furthermore, the choice of biomarker for immunological readout is often dictated by the nature of the vaccine, rather than biologically useful clinical response.

The type of immunological monitoring carried out is largely dictated by the nature of the antigen used for vaccination. Each assay has its own merits and it is likely that no single assay will provide a conclusive correlation with clinical outcome. In general, assays may be divided into two groups; those that are HLA restricted and those that are not. TheH LA-restricted assays show exquisite specificity, and often high sensitivity, but are restricted to particular individuals. M oreover, these methods do not allow the examination of 'epitope spreading' [36]. This phenomenon should occur if a specific immune response against a particular antigen leads to the destruction of a tumor. Therefore, it follows that more antigens will be presented to the immune system and, hence, the immune reaction is effectively amplified.

Alternatively, assays that are not H LA-specific are often more applicable to a large patient population, but suffer from lower sensitivity and are difficult to interpret. Furthermore, the choice of possible biomarkers is huge and some degree of educated choice must be employed to avoid simply collecting large amounts of data. Techniques such as gene arrays are exciting new technologies. However, the enormous amount of data that they produce requires the development of new bioinformatic methodologies to allow meaningful conclusions to be drawn. Similarly, proteomic data, such as from mass spectroscopic approaches, is now becoming more easily accessible. This represents a massive increase in our knowledge of protein expression during disease, but again successful data mining requires the development of new techniques. A summary of some of the major immune monitoring techniques available is given in Table 1.

Using the O nyvax (London, UK) whole-cell vaccine as an example (Box 2), a large amount of data were collected using a variety of multiparametric techniques (Table 2). As described previously, PSA and time to disease progression are useful clinical biomarkers, and both of these were correlated with each recorded variable in turn. No significant trends could be discovered. There fore, an artificial neural network analysis (AN N) was employed that could discern patterns within the data set that were not immediately apparent using classical statistical analysis.

\section{Multiparametric data analysis}

Multilayer perceptron (M LP) artificial neural networks (ANNS) (Figure 1) have been used to develop predictivenumerical models that may discriminate between responders and nonresponders to vaccination based on a range of immunological markers [28]. This approach has led to the development of diagnostic models that have good predictive performance with high specificity and sensitivity for blind data. In turn, these models have been interrogated using sensitivity analysis to determine the relative importance of these discriminatory immunological factors. This step allows both the elimination of noise and redundancy in the model and, with redevelopment, increases diagnostic precision. Application of 'dummy' values to the models has been used to generate 'response surfaces' for the key markers, indicating whether they have a positive influence and whether the marker is discriminatory or cofactorial. By conducting random sample crossvalidation approaches, where multiple models are tested based on different randomly extracted subsets for training and testing, measures of confidence can be determined, both for biomarkers and predictions [37]. Finally, assessment of the predictions for individuals within the population provides an indication of how characteristic they were of the responder/nonresponder groups. 
Table 1. Common methodologies used in immunological profiling .

\section{Assay}

ELISA

ELISPOT

ELISPOT

SEREX

Western blot

Immuno-

histochemistry

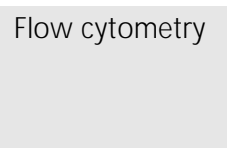

Tetramers

Proliferation

CTL assay

Limiting

dilution assay

Delayed-type

hypersensitivity
Description and applicability

\section{Detects}

ELISA is a 'workhorse' technique, suited to detection of antibody responses to a particular target or to the detection of proteins. In both situations, either the antigen or the antibody is immobilized onto the plate and detection can be achieved using a number of methodologies ranging from colorimetric to fluorescent. There have been numerous refinements of this method, such as the CELISA, in which cells, rather than antigen are used as the target.

This is an extension of the ELISA in which cells are incubated on a membrane containing a capture antibody for a particular cytokine. Once the cells are removed, the membrane is developed using a detection antibody and spots form if a cell, found at that location, produced the cytokine of interest. This is an extremely sensitive technique. How ever, this also makes it inappropriate for all situations due to background level concerns.

Essentially, this is a refined Western blot that allows the identification of antibody specificities by using expression libraries which may then be amplified and sequenced to reveal their identity. This is a very powerful technique, but is extremely labor intensive.

The western blot uses antibodies to identify particular proteins present after gel electrophoresis. The main limitation of the technique is that proteins are generally fully denatured after SDS-PAGE and so many antibodies cannot be used for detection due to the loss of protein tertiary structure.

Coupled with FACS, IHC allows for a full phenotype of a sample to be examined. IHC uses antibodies to identify proteins on slides, either in the form of sections or cytospins, and thus allows data to be gathered regarding the distribution of an antigen. Unlike FACS, it is usually not quantitative. However, it complements FACS because it allows the relationship between cells to be examined.

Flow cytometry, and the availability of monoclonal antibodies, have made the monitoring of specific antigens routine. The assay can be made quantitative by using reference calibration beads and thus enumerate the number of molecules per cell. An extension of the technique is cell sorting, which allows extremely pure cell fractions to be isolated and further analyzed.

Four M HC molecules, loaded with a particular peptide, are fluorescently tagged. If a T-cell carries a receptor of the correct specificity, it will be detectable by flow-cytometry. M ost usually, tetramers are built around MHC class I antigens. However, some class II molecules have recently become available. With the notable exception of viral responses, tetramer levels are usually very low.

Traditionally, proliferation is taken as indicative of a CD4 helper T-cell response, since the CD8 T-cell does not divide to the same degree. In order for such assays to work, antigen processing must be allowed to occur. Therefore, these assays are usually carried out with whole splenocytes (mice), DC and PBL (human) or even in whole blood. Detection may be by use of radio-isotope, fluorescence or even flow cytometry when bromodeoxyuridine is used as a substrate.

Detection of CD8 cytotoxic T-lymphocyte activity is usually achieved using target lysis assays. These can either be radioactively-labeled targets (for example, $\mathrm{Cr}^{51}$ ) or fluorescent detection of protein (for example, LDH). In both cases, the amount of substrate released is proportional to the amount of targets killed by the CTL. Targets are usually M HC-matched, although targets specific for NK cells, for example, can add further refinement.

An extension of the CTL assay is to include a titration of the amount of effectors present in the system. This allows the enumeration of the number of CTL precursors present in a system before and after vaccination.

This is a useful clinical assay that can be used to detect the presence of a cellular immune response to a particular antigen before and after vaccination. Protein is injected into the skin and the size of the inflammation is proportional to the magnitude of the response. This is particularly appropriate for antigens such as BCG.
Antibody responses and/or protein detection

T-cell responses

Antibody responses

Antibody responses

Cellular immune responses

Cellular immune responses

T-cell responses

T-cell responses

T-cell responses

T-cell responses

Cellular immune responses

2D: Two-dimensional; BCG: Bacillus Calmette-Guerin; CBA: Cytometric bead array; CELISA: Competitive enzyme-linked immunosorbent assay; CTL: Cytotoxic T-lymphocyte; DC: Dendritic cell; ELISA: Enzyme-linked immunosorbent assay; FACS: Fluorescence activated cell sorting; IEF: Isoelectric focusing; IHC: Immunohistochemistry; LDH: Lactate dehydrogenase; MHC: Major histocompatibility complex; NK: Natural killer; PBL: Peripheral blood lymphocytes; PCR: Polymerase chain reaction; qPCR: Quantitative polymerase chain reaction; SDS-PAGE: Sodium dodecyl (lauryl) sulfatepolyacrylamide gel electrophoresis; SELDI: Surface-enhanced laser desorption/ionization; TCR: T-cell receptor; $\mathrm{T}_{H}$ : T-helper. 


\section{Table 1. Common methodologies used in immunological proffling (cont.).}

\begin{tabular}{|c|c|c|}
\hline Assay & Description and applicability & Detects \\
\hline $\begin{array}{l}\text { Cytokine } \\
\text { release }\end{array}$ & $\begin{array}{l}\text { Cytokines can be roughly divided into } \mathrm{T}_{H} 1 \text { and } \mathrm{T}_{\mathrm{H}} 2 \text { based on their biological function. } \mathrm{T}_{\mathrm{H}} 1 \text { responses } \\
\text { are largely cytotoxic and } \mathrm{T}_{\mathrm{H}} 2 \text { responses are nonlytic. Therefore, monitoring of gross cytokine levels } \\
\text { can be used to determine the type of immunological response occurring. A number of techniques } \\
\text { are available for this and include ELISA, ELISPOT and CBA, in which flow cytometry is used for } \\
\text { quantitative measurement. }\end{array}$ & $\begin{array}{l}\text { T-cell } \\
\text { responses }\end{array}$ \\
\hline PCR & $\begin{array}{l}\text { The PCR has exquisite sensitivity and, depending on the substrate used, can be used for the } \\
\text { measurement of rare molecules or ones for which no antibody exists. Furthermore, it has recently } \\
\text { been made quantitative and thus can be used for monitoring responses over time. }\end{array}$ & $\begin{array}{l}\text { Cellular } \\
\text { immune } \\
\text { responses }\end{array}$ \\
\hline TCR usage & $\begin{array}{l}\text { PCR analysis of } \mathrm{V} \beta \text { genes, present in the T-cell receptor, can show if an immune response is } \\
\text { becoming clonal, and therefore, less variable. Such a response would be expected if a strong T-cell } \\
\text { response has been induced. }\end{array}$ & $\begin{array}{l}\text { T-cell } \\
\text { responses }\end{array}$ \\
\hline Gene array & $\begin{array}{l}\text { The recent sequencing of the human genome has allowed the development of gene arrays which } \\
\text { can display almost the entire gamut of mRNAs generated by a cell. By hybridizing nucleic acids from } \\
\text { a patient onto these arrays, it is possible to define the exact nature of a response with great } \\
\text { accuracy. How ever, one major caveat is that these profiles are not fully quantitative and usually need } \\
\text { a second technique, such as qPCR to confirm the result. Furthermore, the large amounts of data } \\
\text { generated from these assays requires extensive bioinformatics mining. }\end{array}$ & $\begin{array}{l}\text { Cellular } \\
\text { immune } \\
\text { responses }\end{array}$ \\
\hline $\begin{array}{l}\text { 2D gel } \\
\text { electrophoresis }\end{array}$ & $\begin{array}{l}\text { The 2D gel uses a separation based on charge (SDS-PAGE) followed by a charge-based selection } \\
\text { (IEF). This allows for a very high resolution of protein expression. Historically, the major limitation of } \\
\text { the technique has been one of resolution, although recent systems have made this much more } \\
\text { feasible. Software advances have also made gel comparison much more practical. }\end{array}$ & $\begin{array}{l}\text { Antibody } \\
\text { and cellular } \\
\text { responses }\end{array}$ \\
\hline SELDI & $\begin{array}{l}\text { This is essentially a form of mass spectroscopy, in which proteins are bound to a gold chip using a } \\
\text { variety of matrices. A laser ionises these proteins which may then be detected by mass spectroscopy. } \\
\text { SELDI is unusual in that several different profiles may be generated, depending on the nature of the } \\
\text { chip surface chemistry and so it is imperative that similar chemistries are used before comparison } \\
\text { is made. }\end{array}$ & $\begin{array}{l}\text { Antibody } \\
\text { and cellular } \\
\text { responses }\end{array}$ \\
\hline $\begin{array}{l}\text { Electrospray } \\
\text { mass- } \\
\text { spectroscopy }\end{array}$ & $\begin{array}{l}\text { Recent advances in mass spectroscopy have improved both resolution and identification capabilities. } \\
\text { When coupled with data from the human genome project, it is now possible to identify protein } \\
\text { fragments with great accuracy. }\end{array}$ & $\begin{array}{l}\text { Antibody } \\
\text { and cellular } \\
\text { responses }\end{array}$ \\
\hline
\end{tabular}

2D: Two-dimensional; BCG: Bacillus Calmette-Guerin; CBA: Cytometric bead array; CELISA: Competitive enzyme-linked immunosorbent assay; CTL: Cytotoxic T-lymphocyte; DC: Dendritic cell; ELISA: Enzyme-linked immunosorbent assay; FACS: Fluorescence activated cell sorting; IEF: Isoelectric focusing; IHC: Immunohistochemistry; LDH: Lactate dehydrogenase; MHC: Major histocompatibility complex; NK: Natural killer; PBL: Peripheral blood lymphocytes; PCR: Polymerase chain reaction; qPCR: Quantitative polymerase chain reaction; SDS-PAGE: Sodium dodecyl (lauryl) sulfatepolyacrylamide gel electrophoresis; SELDI: Surface-enhanced laser desorption/ionization; TCR: T-cell receptor; TH: T-helper.

In the first instance, these models have been developed on a relatively small data set. H owever, further development of the model by the addition of newly available data increases the confidence in predicted values and biomarkers identified. UItimately, it should be possible to predict clinical outcome with great precision and achievea greater understanding of the biological system.

\section{Expert commentary}

The evidence for immunological activity against cancer is compelling. In particular, Dunn and colleagues [38] demonstrated that mice lacking the interferon- $\gamma$ receptor spontaneously developed tumors, suggesting that the immune system has a normal anticancer homeostatic function. More recent clinical data from Rosenberg's group, using T-cell transfer, shows remarkable clinical efficacy [39]. H owever, as yet, numbers remain small, and it is questionable that this method could be scaled up for widespread clinical use. There may be a difference between the development of a tumor versus recurrence in terms of immune surveillance. The former would be relevant to the control of cancer in the population and the latter to the development of therapeutic strategies.

$\mathrm{H}$ istorically, most biomarkers in clinical use are defined as either being related to the disease process, for example PSA, or as being involved in the putative mechanism of vaccine efficacy. Particularly in the latter case, the evidence for one particular immunological mechanism being the sole effector function is variable. Clearly T-cell immunity is important, as evidenced by the experiments outlined above. H owever, it remains 


\section{Box 2. Onyvax clinical trial.}

Allogeneic whole-cell vaccination is a viable immunotherapy option for prostate cancer patients. The principle is that replication incompetent prostate tumor cell lines are used as a vaccine in a nontissue-matched manner. Consequently, the immune system sees the vaccine as a 'danger signal' [49] and attacks it, by virtue of its nonhuman leukocyte antigen (HLA) match. In so doing, the tumor cells are broken down and presented by dendritic cells (DC) to the immune system. It is well established that many tumors share antigens to some degree, for example, melanoma antigen gene (M AGE), New York esophageal squamous cell carcinoma 1 (NY-ESO1) and mucin 1 (MUC1), and therefore it is likely that the very presentation of the vaccine to the patient's ow $n$ immune system may lead to the generation of an immune reaction specific for antigens present on the individuals own tumor. Onyvax has carried out both Phase I and II trials which have clearly demonstrated clinical benefit of this approach. How ever, given the very nature of this multicomponent vaccine, the precise nature of the biologically relevant antigens is unknown. Indeed, it is likely that important antigens differ between patients, and it is even possible that immunodominant antigens may vary within the same patient over time. Therefore, immune monitoring of these trials is particularly challenging and must, by definition, include multiple parameters. In a recent Phase lla trial [28], Onyvax produced a database of 20,000 data points, encompassing some 36 variables (Table 2). The correlation of these multiple parameters is complex and cannot be simplified into a two-dimensional model. To address this, Onyvax have used an artificial neural network (ANN) to both identify and then rank biomarkers that show correlation with immune response. Consequently, no attempt is made to prioritise markers before analysis begins, rather multiple parameters are collected and an ANN is used to rank them. In this way, bias is avoided and true relationships can be discovered. In the authors' experience, it is highly unlikely that a simple linear relationship between a single variable and clinical outcome exists. It is only after the use of ANN's that complex biological relationships can be correlated with disease progression.

unclear if this is the only mechanism at play. Indeed, it seems unlikely that this is so, given the variable major histocompatibility complex (M HC) expression of tumors and their varying degrees of immunogenicity.

Therefore, a more empirical approach has been proposed in which no predictions are made as to the nature of the immune response that may be generated and multiple parameters are recorded. In this way, 'immunological profiles' can be built up and then correlated with either clinical outcome or more conventional biomarkers. $\mathrm{H}$ ence, the use of large numbers of biomarkers becomes one of quantity rather than quality. It is still likely that many of these analytes are indeed involved in the immune response, but by removing the relatively narrow range of parameters normally collected, we ensure that almost all immunological mechanisms are measured. It is unlikely that any one single analyte will show complete correlation with clinical response, but, when used in combination with a large number of variables, patterns begin to emerge.

\section{Table 2. Immune parameters recorded during the Onyvax Phase II trial*.}

\begin{tabular}{|c|c|}
\hline Variable & Description \\
\hline $\begin{array}{l}\text { Cell surface } \\
\text { markers }\end{array}$ & $\begin{array}{l}\text { A set of } 8 \text { antigens, in various combinations, were monitored throughout the course of the trial to detect any gross } \\
\text { imbalances in immunity and to ensure that patients maintained a viable immune system during the course of } \\
\text { treatment. } M \text { arkers recorded were CD3, CD4, CD8, CD28, CD38, CD45RA, CD45RO, and CD62L. }\end{array}$ \\
\hline Proliferation & $\begin{array}{l}\text { Specific proliferation to the vaccinating antigens was recorded using a flow cytometric approach. This assay is } \\
\text { considerably more complex than normal proliferations because cell lysate and whole blood must be used to avoid } \\
\text { mixed lymphocyte reactions and allow antigen processing to occur, respectively. Reactivity to all three cell lines used in } \\
\text { the vaccine was recorded, as well as positive (mitogen) and negative (medium) controls. }\end{array}$ \\
\hline $\begin{array}{l}\text { Protein } \\
\text { cytokine } \\
\text { levels }\end{array}$ & $\begin{array}{l}\text { PBL were collected at every clinic visit. These were then maximally stimulated using calcium ionophore to establish the } \\
\text { maximum amount of cytokine that they could produce. This was then measured using CBA, which is a fully } \\
\text { quantitative flow cytometric capture assay. Thus, a quantitative time course was created for the entire clinical trial. The } \\
\text { cytokines measured were IFN- } \gamma, \text { IL-2, }-4,-5,-10 \text { and TNF- } \alpha \text {. }\end{array}$ \\
\hline $\begin{array}{l}\text { mRNA } \\
\text { cytokine } \\
\text { levels }\end{array}$ & $\begin{array}{l}\text { The same samples used for protein cytokine analysis were also converted into cDNA after stimulation. A fully } \\
\text { quantitative PCR technology was then employed which was able to show the number of copies of each target gene } \\
\text { transcribed over time. By expressing this as a ratio against accurate CDNA amounts, it was possible to examine } \\
\text { changes over time. Before this advance, such analysis was impossible. The cytokines measured were IFN- } \gamma, \mathrm{IL}-2,-4 \text {, } \\
-5,-10 \text { and TNF- } \alpha \text {. }\end{array}$ \\
\hline PSA values & $\begin{array}{l}\text { PSA levels were routinely measured and then converted into a PSA velocity. Using this methodology, trends are more } \\
\text { easily detected and erroneous data-points are more apparent. }\end{array}$ \\
\hline
\end{tabular}

CBA: Cytometric bead array; IFN: Interferon; IL: Interleukin; PBL: Peripheral blood monocytes; PCR: Polymerase chain reaction; PSA: Prostate-specific antigen; TNF: Tumor necrosis factor.

*Data obtained from [28]. 


\section{Figure 1. The multi layer perceptron.}

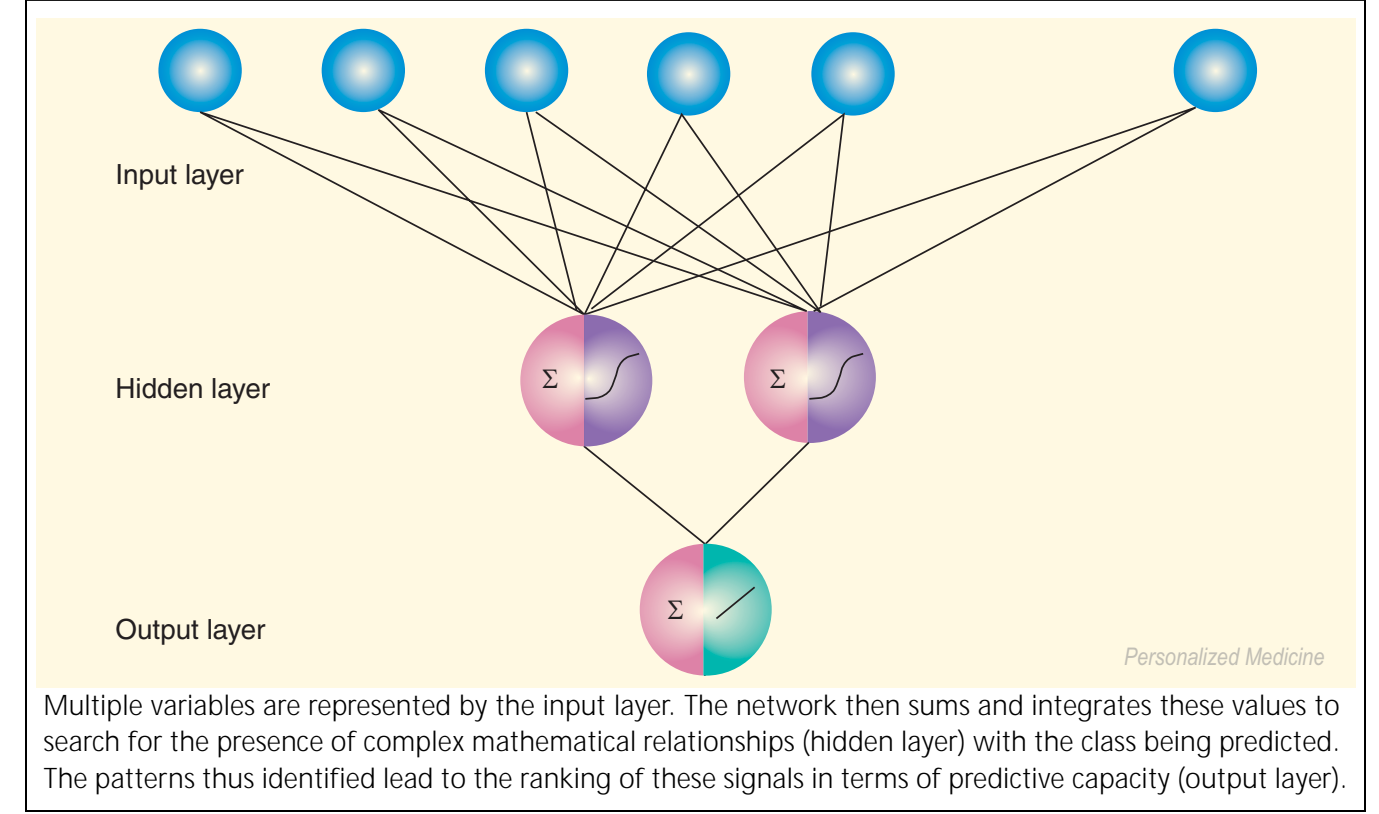

As a consequence of this approach, data analysis becomes of crucial importance. Such large data sets cannot be easily compared using conventional statistics, largely due to colinearity, nonlinearity and redundancy parameters. Appropriate analysis requires the use of more powerful computational techniques. Neural networks have been widely shown to provide sufficient computational power to achieve this [40].

Therefore, these data raise the fundamental question of, do weneed 'definitive' markers or just a large number of relatively innocuous molecules to give an overall profile? The authors studies indicate that both may be true. If a truly disease specific biomarker can be isolated, this is of obvious clinical benefit. H owever, these are very rare and unlikely to be isolated for every disease. Furthermore, it remains to be demonstrated that these levels will alter during successful treatment. Therefore, by using immunological profiles in tandem, it should be possible to identify clinically responding patients at an early stage. As AN N's develop, it is quite likely that predictions of clinical outcome can be made, thus sparing patients treatment regimens that may not be efficacious. If this can be validated in a large-scale trial, it may greatly increase the probability of a good clinical outcome for the patient and may even be used predictively by assessing the patient's 'immune profile' to conduct a risk assessment for the patient before any treatment is administered.

The primary aim of biomarker research is to differentiate responding and nonresponding patients as early as possible. This is particularly true for immunotherapies since it is well established that immune suppression begins as disease advances. The ben efit to patients is clearly paramount and the ability to identify productive antitumor responses at an early stage would allow the optimal therapy to be identified for a particular individual. A further advantage that arises from biomarker research is that, when used in a predictive modality, it is likely that these will shorten vaccine development times. This has obvious ramifications for the biotechnology industry, since clinical trials run over shorter time periods will reduce costs and increase the throughput of new strategies into the clinic.

\section{Highlights}

- Biomarkers are useful surrogates for clinical parameters and validated molecules can greatly shorten clinical development times for new drugs.

- Immune mechanisms are increasingly being used to combat cancer.

- The field of cancer vaccines is large and varied, consisting of multiple strategies.

- In many cases the type of vaccine employed dictates the immunological monitoring approach used.

- Use of multiparametric data capture combined with advanced bioinformatic techniques can produce new and powerful molecular profiles which have utility as biomarkers. 
Bibliography

Papers of special note have been highlighted as

either of interest $(\bullet)$ or of considerableinterest $(\cdot \bullet)$ to readers.

1. Collette L, Burzykowski T, Carroll KJ et al.: Is prostate-specific antigen a valid surrogate end point for survival in hormonally treated patients with metastatic prostate cancer? Joint research of the European $O$ rganisation for Research and Treatment of $\mathrm{C}$ ancer, the Limburgs Universitair Centrum. J. Clin. Oncol. 23(25), 6139-6148 (2005).

2. Small EJ, Roach $M$ : Prostate-specific antigen in prostate cancer: a case study in the development of a tumor marker to monitor recurrence and assess response. Semin. Oncol. 29(3), 264-273 (2002).

3. Koenig W: Predicting risk and treatment benefit in atherosclerosis: the role of C-reactive protein. Int. J. Cardiol. 98(2), 199-206 (2005).

4. Segal R, Lukka H, Klotz LH et al.: Surveillance programs for early stage nonseminomatous testicular cancer: a practice guideline. Can. J. U rol. 8(1), 1184-1192 (2001).

5. Lewis S, M enon U: Screening for ovarian cancer. Expert. Rev. AntiCancer Ther. 3(1), 55-62 (2003).

6. $\mathrm{Ko} A H, \mathrm{H}$ wang J, Venook AP et al.: Serum CA 19- 9 response as a surrogate for clinical outcome in patients receiving fixed-dose rate gemcitabine for advanced pancreatic cancer. Br. J. Cancer. 93(2), 195-199 (2005).

7. Bendardaf R, Lamlum H, Pyrhonen S: Prognostic and predictive molecular markers in colorectal carcinoma. AntiC ancer Res. 24(4), 2519-2530 (2004).

8. Cilloni D, Saglio G: W T 1 as a universal marker for minimal residual disease detection and quantification in myeloid leukemias and in myelodysplastic syndrome. Acta H aematol. 112(1-2), 79-84 (2004).

9. Kabbinavar FF, $\mathrm{H}$ ambleton J, $M$ ass RD et al.: Combined analysis of efficacy: the addition of bevacizumab to fluorouracil/leucovorin improves survival for patients with metastatic colorectal cancer. J. Clin. O ncol. 23(16), 3706-3712 (2005).

10. Perez-Soler R: The role of erlotinib (Tarceva, OSI 774) in the treatment of non-small cell lung cancer. Clin. Cancer Res. 10(12 Pt 2), S4238-S4240 (2004).

11. Powell DJ Jr, Parker LL, Rosenberg SA: Large-scale depletion of CD 25+ regulatory $\mathrm{T}$-cells from patient leukapheresis samples. J. Immunother. 28(4), 403-411 (2005).

12. Rock KL, Shen L: Cross-presentation: underlying mechanisms and role in immune surveillance. Immunol. Rev. 207, 166-183 (2005).

13. Slingluff $C L J$, Petroni $G R$, Yamshchikov GV et al.: Immunologic and clinical outcomes of vaccination with a multiepitope melanoma peptide vaccine plus low-dose interleukin-2 administered either concurrently or on a delayed schedule. J. Clin. O ncol. 22(22), 4474-4485 (2004).

14. Muderspach $\mathrm{L}$, W ilczynski $\mathrm{S}$, Roman $\mathrm{L}$ et al.: A Phase I trial of a human papillomavirus (H PV) peptide vaccine for women with high-grade cervical and vulvar intraepithelial neoplasia who are H PV 16 positive. Clin. Cancer Res 6(9), 3406-3416 (2000).

15. Slingluff $C L J$ Jr, Petroni $G R$, Yamshchikov GV et al.: C linical and immunologic results of a randomized Phase II trial of vaccination using four melanoma peptides either administered in granulocytemacrophage colony-stimulating factor in adjuvant or pulsed on dendritic cells. J. Clin. Oncol. 21(21), 4016-4026 (2003).

16. M iller AM, O zenci $V$, Kiessling $R$ et al.: Immune monitoring in a Phase 1 trial of a PSA D N A vaccine in patients with hormone refractory prostate cancer. J. Immunother. 28(4), 389-395 (2005).

17. Stevenson FK, Rice J, 0 ttensmeier $\mathrm{CH}$ et al:: D N A fusion gene vaccines against cancer: from the laboratory to the clinic. Immunol. Rev. 199, 156-180 (2004).

18. Liu M, Acres B, Balloul JM et al.: Genebased vaccines and immunotherapeutics. Proc. Natl Acad. Sci. U SA 101(Suppl. 2), 14567-14571 (2004).

19. Davis ID, Chen W, Jackson $\mathrm{H}$ et al.: Recombinant N Y-ESO -1 protein with ISC O M ATRIX adjuvant induces broad integrated antibody and CD $4^{+}$and $C D 8^{+}$ $\mathrm{T}$-cell responses in humans. Proc. N atl Acad. Sci. USA 101(29), 10697-10702 (2004).

20. Burch PA, Croghan GA, G astineau DA et al.: Immunotherapy (APC 8015, Provenge) targeting prostatic acid phosphatase can induce durable remission of metastatic androgen-independent prostate cancer: a Phase 2 trial. Prostate 60(3), 197-204 (2004).

21. Small EJ, Fratesi P, Reese D M et al.: Immunotherapy of hormone refractory prostate cancer with antigen-loaded dendritic cells. J. Clin. O ncol. 18(23), 3894-3903 (2000).

22. Su Z, D annull J, H eiser $A$ et al.: Immunological and clinical responses in metastatic renal cancer patients vaccinated with tumor RN A-transfected dendritic cells. Cancer Res.63(9), 2127-2133 (2003).
23. Dillman RO, Beutel LD, Barth N M et al.: Irradiated cells from autologous tumor cell lines as patient-specific vaccine therapy in 125 patients with metastatic cancer: induction of delayed-typehypersensitivity to autologous tumor is associated with improved survival. Cancer Biother. Radiopharm. 17(1), 51-66 (2002).

24. M oiseyenko VM, D anilov AO, Baldueva IA et al.: Phase I/II trial of gene therapy with autologous tumor cells modified with tag7/PG RP-S gene in patients with disseminated solid tumors: miscellaneous tumors. Ann. Oncol. 16(1), 162-168 (2005).

25. Dillman $\mathrm{R}$, Barth $\mathrm{N}$, Vandermolen $\mathrm{L}$ et al.: Autologous tumor cell line-derived vaccine for patient-specific treatment of advanced renal cell carcinoma. Cancer Biother. Radiopharm. 19(5), 570-580 (2004).

26. Coulie PG, Karanikas V, Lurquin C et al.: Cytolytic T-cell responses of cancer patients vaccinated with a M AGE antigen. Immunol. Rev. 188, 33-42 (2002).

27. Gilewski T, Adluri S, Ragupathi $G$ et al.: Vaccination of high-risk breast cancer patients with mucin-1 (M U C 1) keyhole limpet hemocyanin conjugate plus Q S-21. Clin. Cancer Res 6(5), 1693-1701 (2000).

28. M ichael A, Ball G, Q uatan N et al.: D elayed disease progression after allogeneic cell vaccination in hormone-resistant prostate cancer and correlation with immunologic variables. Clin. Cancer Res 11(12), 4469-4478 (2005).

- First recorded use of multiparametric analysis correlating with clinical response.

29. H sueh EC, Essner R, Foshag $L$ J et al.: Prolonged survival after complete resection of disseminated melanoma and active immunotherapy with a therapeutic cancer vaccine. J. Clin. O ncol. 20(23), 4549-4554 (2002).

- Phase II data using allogeneic whole cell vaccine in melanoma.

30. Agarwala SS, N euberg D, Park Y et al.: $M$ ature results of a Phase III randomized trial of bacillus $C$ almette-Guerin (BCG) versus observation and $B C G$ plus dacarbazine versus BCG in the adjuvant therapy of American Joint Committee on Cancer Stage I-III M elanoma (E1673): a trial of the Eastern. Cancer 100(8), 1692-1698 (2004).

31. Cascinelli N, RumkeP, M acKieR et al.: The significance of conversion of skin reactivity to efficacy of bacillus Calmette-Guerin (BCG) vaccinations given immediately after radical surgery in stage II melanoma 
patients. Cancer Immunol. Immunother. 28(4), 282-286 (1989).

32. Sondak VK, Liu PY, Tuthill RJ et al.: Adjuvant immunotherapy of resected, intermediate-thickness, node-negative melanoma with an allogeneic tumor vaccine: overall results of a randomized trial of the Southwest O ncology G roup. J. Clin. O ncol. 20(8), 2058-2066 (2002).

33. Sosman JA, Sondak VK: M elacine: an allogeneic melanoma tumor cell lysate vaccine. Expert Rev. Vaccines 2(3), 353-368 (2003).

34. Germeau C, M a W, Schiavetti F et al.: H igh frequency of antitumor $\mathrm{T}$-cells in the blood of melanoma patients before and after vaccination with tumor antigens. J. Exp. M ed. 201(2), 241-248 (2005).

35. Lurquin $C$, Lethe B, D e Plaen $E$ et al.: Contrasting frequencies of antitumor and antivaccine $T$-cells in metastases of $a$ melanoma patient vaccinated with a M AGE tumor antigen. J. Exp. M ed. 201(2), 249-257 (2005).

- Variation in the specificity of T-cell response after specific peptide vaccination.

36. Disis M L, Goodell V, Schiffman K et al.: H umoral epitope-spreading following immunization with a HER-2/neu peptide based vaccine in cancer patients. J. Clin. Immunol. 24(5), 571-578 (2004).

37. Lancashire L, M ian S, Ellis I et al.: Current developments in the analysis of proteomic data: artificial neural network data mining techniques for the identification of proteomic biomarkers related to breast cancer. Current Proteomics 2(1), 15-29 (2005).

- D evelopment of artificial neural network (AN N) models in mass spectroscopic studies.

38. Dunn GP, Bruce AT, Ikeda H et al.: Cancer immunoediting: from immunosurveillance to tumor escape. N ature Immunol. 3(11), 991-998 (2002).

- Strong evidence for immune control of tumors in vivo.

39. Rosenberg SA, D udley M E: C ancer regression in patients with metastatic melanoma after the transfer of autologous antitumor lymphocytes. Proc. N atl Acad. Sci. USA 101(Suppl. 2), 14639-14645 (2004).

40. Ball G, M ian S, H olding F et al.: An integrated approach utilizing artificial neural networks and SELDI mass spectrometry for the classification of human tumors and rapid identification of potential biomarkers. Bioinformatics 18(3), 395-404 (2002).

- $\quad$ AN N and tumor biomarker studies.

41. Petrylak D P: Future directions in the treatment of androgen-independent prostate cancer, U rology 65(Suppl. 6), 8-12 (2005).

42. Burnet FM: The concept of immunological surveillance. Prog. Exp. Tumor Res. 13, 1-27 (1970).

43. Burnet FM: Immunological aspects of malignant disease. Lancet 1(7501),

1171-1174 (1967).

44. W iemann B, Starnes C O : Coley's toxins, tumor necrosis factor and cancer research: a historical perspective. Pharmacol.T her. 64(3), 529-564 (1994).
45. Collette L, Burzykowski T, Carroll KJ et al.: Is prostate-specific antigen a valid surrogate end point for survival in hormonally treated patients with metastatic prostate cancer? Joint research of the European 0 rganisation for Research and Treatment of $C$ ancer, the Limburgs Universitair Centrum. J. Clin. Oncol. 23(25), 6139-6148 (2005).

46. Scher HI, Eisenberger M, D 'Amico AV et al.: Eligibility and outcomes reporting guidelines for clinical trials for patients in the state of a rising prostate-specific antigen: recommendations from the Prostate-Specific Antigen Working Group. J. Clin. 0 ncol. 22(3), 537-556 (2004).

- Highlights that the velocity of PSA rise may be a more important biomarker than absolute PSA levels.

47. US Food and D rug Administration: FD A Public Workshop on Clinical Trial End points in ProstateCancer. FDA, M D, USA (2004).

48. D atta M W, D hir R, D obbin $\mathrm{K}$ et al.: Prostate cancer in patients with screening serum prostate specific antigen values less than $4.0 \mathrm{ng} / \mathrm{dl}$ : results from the co-operative prostate cancer tissue resource. J. U rol. 173(5), 1546-1551 (2005).

49. $M$ atzinger $P$ : The danger model: a renewed Sense of self. Science 296(5566), 301-305 (2002).

Website

101. The CancerVax ${ }^{T M}$ website. www.cancervax.com 